family contacts during the first year of his treatment. Contacts include all the other members of the family, but the inquiry was limited to 'close' family contacts, that is, those who had been living with the index case for at least three months before diagnosis. In fact, $96 \cdot 7$ per cent of the 693 close family contacts had been living with the index case for at least a year.

Examinations were carried out in 693 contacts of the 193 patients treated at home or in the sanatorium; of these, 347 were men and 346 women. The survey of the prevalence of tuberculosis when the index case started treatment was based on 672 contacts. Active tuberculosis was found in 46 ; in another 2 , activity was doubtful; and in 11, tuberculosis was inactive.

Of still more interest was the inquiry into fresh cases of tuberculosis appearing in close family contacts of patients treated either at home or in the sanatorium during the first year of treatment. There were 257 contacts of the 75 patients treated at home, and 275 contacts of the 73 patients in the sanatorium. The two groups were alike in the proportion of men to women, in the kind of home they lived in and in the results of the initial examination; and they were followed up in the same way during the year of the inquiry. During this year, 26 active cases of tuberculosis were discovered in the two groups of contacts ; $9(3.5$ per cent) in the group of contacts of patients treated at home, and $17(6 \cdot 2$ per cent) in the group of contacts of patients treated in the sanatorium.

Of these 26 cases, 15 were less than five years old and 21 less than two years old. The most serious cases all belonged to the group whose index cases were treated in the sanatorium.

Even if encouraging conclusions can be drawn from this study, domiciliary treatment of the open tuberculous patients should be considered an emergency measure only, to be taken when institutional treatment for 3-6 months is impossible because of shortage of beds. Every effort should be made not to send patients home until their sputum has become negative for tubercle bacilli, at least on microscopical examination.

A final conclusion is that the frequency and seriousness of tuberculosis lesions in young contacts are a sufficient justification for systematic prophy lactic treatment of children whenever a sputumpositive patient is newly diagnosed in their household. This treatment should be started immediately and carried out for a minimum period of six months.

\title{
NOISE IN FACTORIES AND ITS CONTROL
}

$\mathrm{N}$ OISE is always associated with the application of power, and the trend towards the use of more powerful machines in industry is making the problem of noise in factories increasingly serious. A survey "Noise in Factories", by A. G. AlderseyWilliams, which has recently been published for the Department of Scientific and Industrial Research (Building Research Station) in the series "Factory Building Studies" (No. 6. Pp. iv +26 . London : H.M. Stationery Office, 1960. 3s. 6d. net), emphasizes that the noise factor should be considered at the earliest stages in the planning of a factory. Choice of the manufacturing method, which largely determines the noise-level and its effect on the occupants of the factory and residents in the area, is the responsibility of the management. Noise at source can be reduced by machine manufacturers in the design of their machines, but where this is not possible, factory engineers can minimize the effect by taking precautions in the installation of the machines, and the building designer can reduce annoyance by siting noisy equip. ment away from other buildings and by the use of enclosures and sound-absorbing roof linings.

The 26-page booklet consists of two parts. Part 1 deals with the properties of sound and explains the terms used, the sources of noise in factories, and the effects of noise. It is pointed out that the most intense noise in factories is generally caused by machine tools and by operations involved in making and handling the product, and it is only when the process is a quiet one that the noise from service equipment, such as fans, pumps and compressors, may be the more intense. Excessive noise will influence the welfare, safety and, indirectly, the efficiency of the occupants of the factory. As the level of noise increases, its effect on people is first annoyance, then interference with communication and, finally, damage to hearing. In addition, since noise is associated with vibration, the performance of delicate equipment in the factory may be upset, and if the noise is transmitted through the factory structure or through the ground to the outside of the factory, it may also cause discomfort.

In Part 2, methods for the reduction of noise and their effectiveness are discussed. The radiation of noise, resonance and damping, and vibration isolation are dealt with first, then noise reduction and lay-out, enclosures to reduce noise, sound-absorbing materials, partial enclosures and screens, and finally ear protection. The most effective way of controlling noist: once it has been generated and radiated is to contain it within an enclosure, a reduction of about $50 \mathrm{db}$. being readily provided by an ordinary building enclosure; but the efficiency of an enclosure depends on its completeness and uniformity, and direct air leaks through a hole or through porous material reduce the efficiency considerably. The build-up of reverberant sound within an enclosure can be controlled by the use of sound-absorbing materials to reduce the amount of sound reflected. The booklet gives a list of absorption coefficients of common sound-absorbing materials and contains illustrations of various sound-absorbing arrangements in factories. For individual ear protection, where operators of noisy machines are exposed to high levels of noise, two forms of commercially available 'ear defenders' are ear plugs and ear muffs. Ear plugs are inexpensive, small and inconspicuous, but may be uncomfortable when first worn. Dry cotton wool is not an effective ear plug. Ear muffs fit over the ears, and though heavier and more expensive than ear plugs, they give greater reduction of noise. In the latest types the seal around the ear is made by a plastic cushion partly filled with liquid, and the muff is very comfortable to wear even for long periods of time.

S. Weintroub 